\title{
DOS NUEVAS ESPECIES DE LA FAMILIA PELTIDIIDAE BOECK, 1873 (COPEPODA: HARPACTICOIDA) DE CUBA
}

\author{
Carlos Varela ${ }^{1}$ y Samuel Gómez ${ }^{2}$ \\ ${ }^{1}$ Department of Marine Biology and Fisheries, Rosenstiel School of Marine and Atmospheric Science, \\ University of Miami, U.S.A. varela06@gmail.com \\ ${ }^{2}$ Universidad Nacional Autónoma de México, Instituto de Ciencias del Mar y Limnología, Unidad \\ Académica Mazatlán. Joel Montes Camarena s/n, 82040, Mazatlán, Sinaloa, México. \\ samuelgomez@ola.icmyl.unam.mx
}

\section{RESUMEN}

Se describen dos especies nuevas de copépodos de la familia Peltidiidae. Una de estas pertenece al género Peltidium Philippi, 1838 y se asocia con el grupo de especies compuesto por P. ovale Thompson I. C. \& Scott A., 1903, P. simplex Nicholls, 1941, P. proximus Varela, 2005 y P. perturbatum Geddes, 1968. Estas especies comparten el exopodito alargado de la pata cinco (P5) en la hembra, lóbulo basoendopodal pobremente desarrollado y la armadura del endopodito de las patas 2-4 (P2-P4). La nueva especie parece ser más similar a $P$. ovale y $P$. simplex, pero difiere en el número de setas/espinas en el exopodito de la P5 de la hembra y en el rostro. La otra especie nueva descrita pertenece al género Eupelte Claus, 1860, tratándose del primer registro de este género para el Mar Caribe. Esta nueva especie de Eupelte parece ser más similar a $E$. gracilis y E. aurulenta Wells y Rao, 1987, pero difiere de ambas en la proporción entre las longitudes de ambos exopoditos de la P1. Esta dos especies se suman a otras de copépodos harpacticoides marinos encontradas recientemente en Cuba, las que han resultado ser nuevos táxones para la ciencia o primeros registros para esta isla.

Palabras clave: Copepoda, Harpacticoida, Peltidiidae, Peltidium, Eupelte.

Title. Two new species of the family Peltidiidae Boeck, 1873 (Copepoda: Harpacticoida) from Cuba.

\section{ABSTRACT}

Two new species of copepods belonging to the Peltidiidae family are herein described. One of these species was attributed to the genus Peltidium and belongs to the group of species composed of P. ovale Thompson I. C. \& Scott A., 1903; P. simplex Nicholls, 1941; P. perturbatum Geddes, 1968; P. proximus Varela, 2005. These species share the elongate female exopodite P5, the poorly-developed baseoendopodal lobe, and the armature formula of endopodite of P2-P4 (with three, four and four setae, respectively). The new species showed to be more similar to $P$. ovale and P. simplex, but differs in the number of setae/spines in the female exopodite P5 (with five setae/spines in the latter two species, but with six elements in the new one), and in the rostrum. The description of a new species of Eupelte is also provided in the present contribution, and is the first record of the genus for the Caribbean Sea. This new species seems to be more closely related to E. gracilis Claus, 1860 and E. aurulenta Wells y Rao, 1987, but differs in the P1 EXP1:EXP2 length ratio. Those two new species are added to several species of marine harpacticoid copepods have been found recently in Cuba. Some of these species turned out to be new to science or new records for Cuba.

Key words: Copepoda, Harpacticoida, Peltidiidae, Peltidium, Eupelte. 


\section{OBJETIVO}

- Describir dos especies nuevas de copépodos pertenecientes a la familia Peltidiidae colectados en aguas cubanas.

\section{INTRODUCCIÓN}

Los integrantes de la familia Peltidiidae poseen un cuerpo deprimido dorsoventralmente y altamente quitinizado. Aunque algunas de las especies de esta familia han sido halladas asociadas a corales, este grupo de copépodos son habitantes comunes de las algas marinas (Boxshall y Halsey, 2004). Para el Golfo de México y el Mar Caribe solo se registran hasta el momento cuatro especies de peltídidos pertenecientes a los géneros Peltidium Philippi, 1838 y Alteutha Baird, 1846 (Varela, 2005 y 2010; Varela et al., 2008 y Gómez y Varela, 2013). Varias colectas de copépodos harpacticoides se realizaron en los años 2009 y 2010 durante expediciones de investigación en las costas suroccidental y norcentral de Cuba, hallándose los copépodos que se describen en este trabajo.

\section{MATERIALES Y MÉTODOS}

Se colectaron macroalgas con la ayuda de SCUBA las cuales se colocaron in situ en una bolsa de polietileno. Posteriormente se añadieron gotas de formalina a la bolsa y el contenido se agitó vigorosamente para colectar los copépodos adheridos. Las muestras fueros tamizadas y los copépodos fueron separados manualmente con la ayuda de pinzas usando un estereomicroscopio. Las observaciones y los dibujos fueron hechos a un espécimen diseccionado completamente usando un microscopio clínico equipado con una cámara clara. Los ejemplares tipo fueron depositados en la Colección Natural Marina del Acuario Nacional de Cuba. La abreviaturas usadas en el texto son: EXP, exopodito; END, endopodito; BENP, basoendopodito, P1, Pata 1; P2, Pata 2; P3, Pata 3; P4, Pata 4 y P5, Pata 5".

\section{RESULTADOS}

Orden Harpacticoida Sars, 1903

Familia Peltidiidae Boeck, 1873

Subfamilia Peltidiinae Claus, 1860

Género Eupelte Claus, 1860

Eupelte cubensis sp. nov.

Figuras 1-3

Descripción del holotipo. Largo máximo $505 \mu \mathrm{m}$, excluyendo el rostro y la rama caudal. Ancho máximo $150 \mu \mathrm{m}$. Cuerpo aplanado dorsoventralmente, débilmente arqueado a lo largo de la línea media, ovoide, sin un complejo patrón de engrosamientos quitinosos. Cefalotórax, rostro y todos los segmentos corporales cubiertos con pelos táctiles distribuidos aleatoriamente. Margen dorsal posterior en todos los segmentos corporales finamente aserrado y con una hilera de pelos táctiles. Rostro rectangular y amplio, no definido en la base, la punta se dobla hacia debajo y hacia adelante. Rama caudal ligeramente más larga que ancha con el borde posterior oblicuo y con cinco setas de diferente longitud. Dos setas en la esquina exterior, dos grupos de largas espínulas aparecen subdistalmente en la cara posterior. Anténula de nueve artejos, los tres primeros con pequeñas espínulas en el borde inferior. Artejo 4 con estetasco.

Antena con basipodito rectangular, dos veces más largo que ancho, con un proceso en la parte ventral de una seta espinulosa en la esquina interna distal. Artejo 1 del endopodito tan largo 

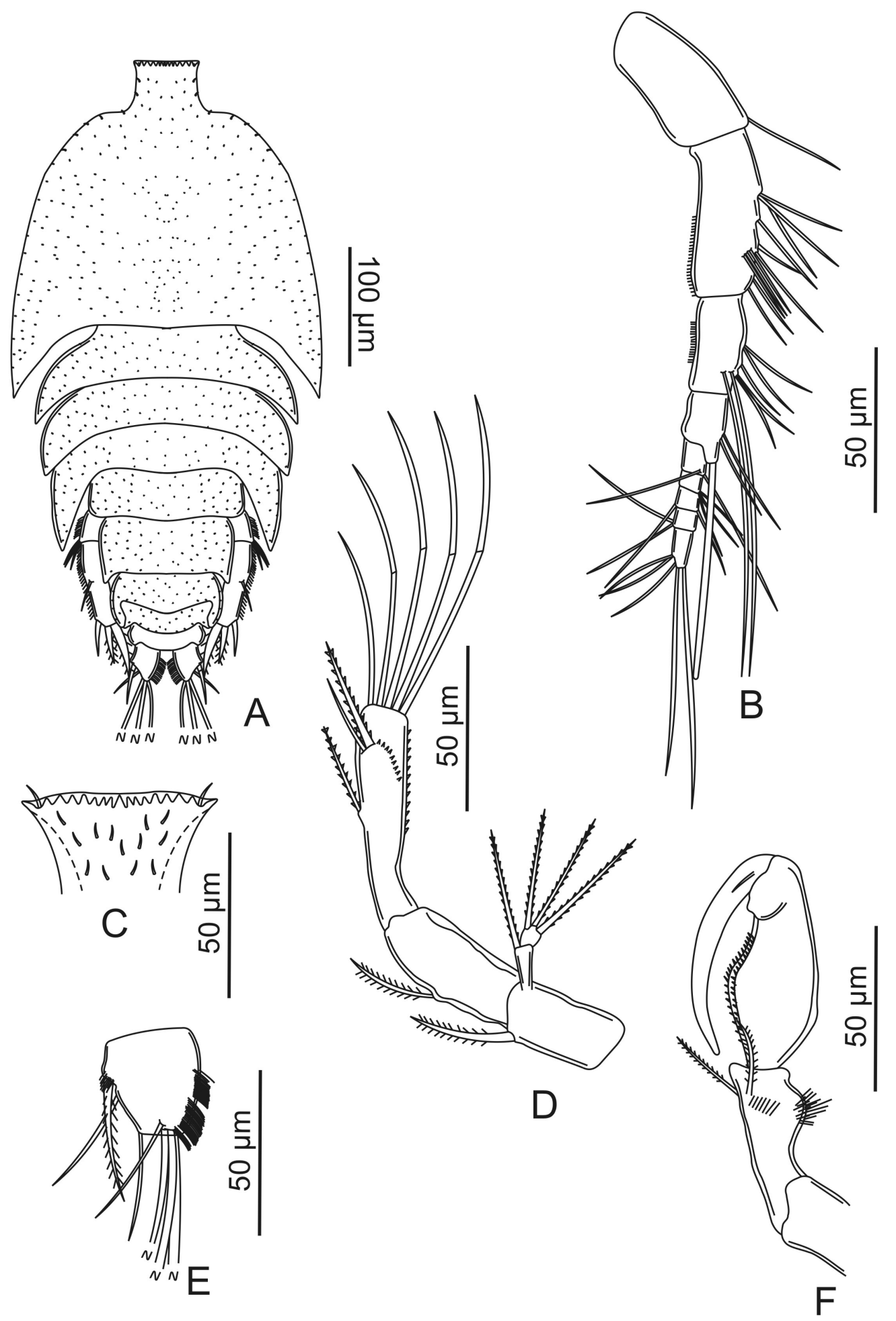


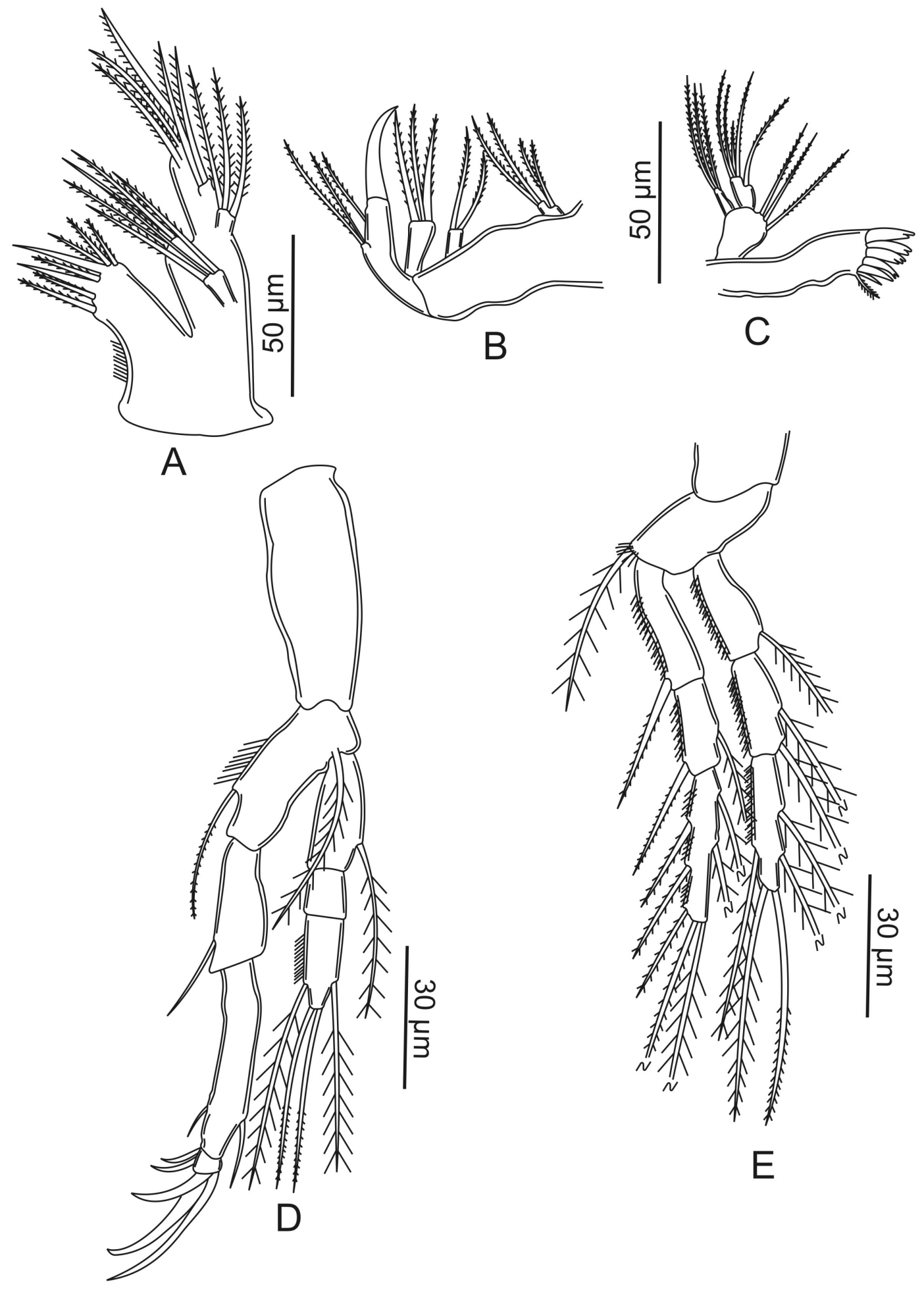

Figura 2. Eupelte cubensis sp. nov. A. Maxílula; B. Maxila; C. Mandíbula; D. Pata 1 y E. Pata 2. 
como el basipodito pero algo alargado y con una seta espinulosa en el medio de su margen distal interno. Artejo dos del endopodito, estrecho y alargado con dos setas espinulosas, una cerca del medio y la otra en el tercio proximal, cinco setas distales, cuatro de ellas geniculadas. Exopodito con dos artejos, el primero con una seta espinulosa y el segundo con tres.

Mandíbula con precoxopodito delgado y alargado con cinco fuertes espinas en su extremo distal, tres de ellas bidentadas y una tridentada, una seta simple y una espinulosa en su borde interno. Endopodito con cinco setas espinulosas y exopodito con tres setas. Maxílula con el artrito del coxopodito bien desarrollado con ocho setas espinulosas distales. Coxopodito con cuatro setas espinulosas y basipodito con tres setas. Endopodito representado por dos largas setas espinulosas y el exopodito por tres setas. Maxila rectangular con tres enditos, el proximal con tres setas espinulosas, el segundo y el tercero con dos y tres respectivamente. Endopodito pequeño con dos setas espinulosas. Endito basal con una fuerte espina terminal unguiforme y dos setas espinulosas.

Maxilípedo prensil. Basipodito delgado y alargado con dos setas espinulosas subdistalmente en el margen ventral. Cara anterior con espínulas, palma inflada proximalmente y ligeramente cóncava en su margen distal externo. Garra curvada con una seta diminuta en su cara posterior.

Pata 1 con coxopodito alargado. Basipodito con una seta espinulosa subapical en el margen interno y otra seta en el margen interior. Exopodito con tres artejos, artejo dos es el más largo de los tres, artejo 1 con una seta y artejo dos con dos setas. Artejo tres corto, casi tan ancho como largo con cinco setas curvadas. Endopodito con dos artejos, artejo uno con una seta y artejo dos con cuatro setas. Patas 2-4, todas con tres artejos en cada rama. El patrón de espinas y setas se muestra a continuación:

\begin{tabular}{lcl}
\hline Pata & Exopodito & Endopodito \\
\hline P1 & $0: 1: 023$ & $1: 221$ \\
P2 & $0: 1: 223$ & $1: 2: 221$ \\
P3 & $0: 1: 323$ & $1: 2: 221$ \\
P4 & $0: 1: 323$ & $1: 2: 221$ \\
\hline
\end{tabular}

Tipos. Holotipo. Hembra no ovígera. CUBA. Colectada cerca del faro de Cayo Palomo, sur de la provincia de Matanzas (22 06' 18" N; 81 28' 29” W); 29.iv. 2010 en macroalgas a $15 \mathrm{~m}$ de profundidad; col. C. Varela. Depositado en la Colección Natural Marina del Acuario Nacional de Cuba ANC.07.2.1.057. Paratipo. Hembra no ovígera. Los mismos datos del holotipo ANC.07.2.1.058

Etimología. La especie fue nombrada cubensis por la isla de Cuba, donde fue colectada.

Comentario. El género Eupelte Claus, 1860, está compuesto actualmente por diez especies, las cuales han sido registradas para latitudes frías y templadas en los Océanos Atlántico y Pacífico. Hicks (1982) presenta el primer análisis del género, pero omite las especies E. simile (Monk, 1941) y E. minuta (Ramírez, 1971). Las especies Eupelte villosa (Brady, 1910) y E. gracilis Claus, 1860, han sido redescritas en varias ocasiones, pero los caracteres diagnósticos en dichas redescripciones rara vez coinciden. Lo cual sugiere que dichas especies tal y como son conocidas en la actualidad son de hecho un complejo de especies (Wells y Rao, 1987 y Dahms, 1992). 

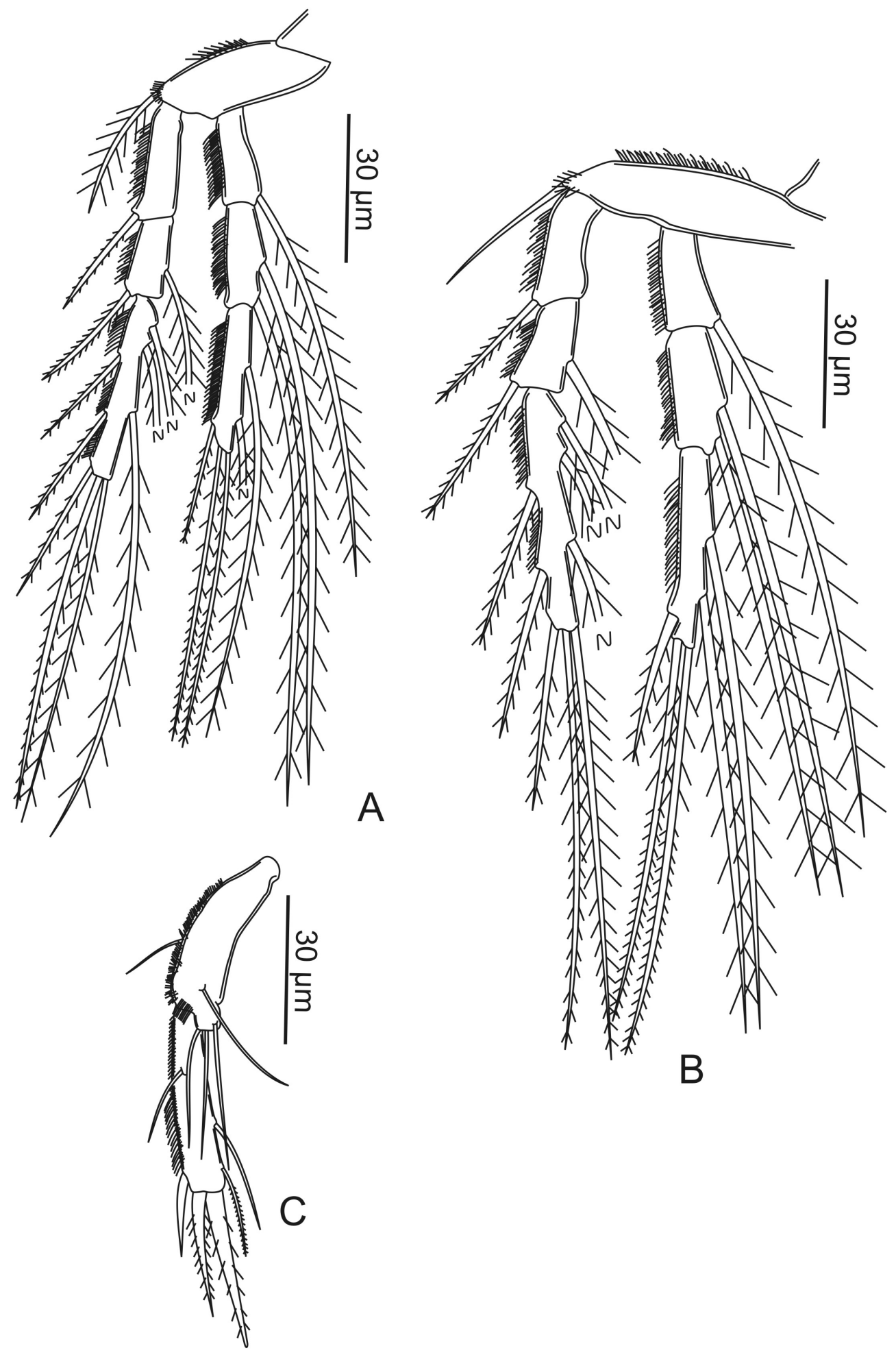

Figura 3. Eupelte cubensis sp. nov. A. Pata 3; B. Pata 4 y C. Pata 5. 
La fórmula de la armadura del EXP P2-P3 (7: 8: 8) y del EXP P1 (1: 4) de E. cubensis, especie nueva, solo es compartido dentro del género, por las especies E. gracilis, E. setacauda Monk, 1941del este del Océano Pacífico; E. aurulenta Wells y Rao, 1987 del Océano Índico y E. acutispinis Zhang y Li, 1976 del oeste del Océano Pacífico. Eupelte cubensis sp. nov. se diferencia de E. gracilis [sensu Pallares (1968)] del Atlántico Sur y de E. acutispinis por tener el END2 de P2-P4 con la fórmula (2: 2: 2), mientras que en E. gracilis [sensu Pallares (1968)] la fórmula es (1: 1: 1) y en E. acutispinis (2: 2: 1). Eupelte cubensis sp. nov. se diferencia de $E$. aurulenta y de E. gracilis [sensu Lang (1948)] del Mar Mediterráneo por la proporción entre la longitud de los dos artejos del EXP P1. En E. cubensis sp. nov. el EXP1 P1 es menos de dos veces la longitud del EXP2 P1 mientras que en E. aurulenta y en E. gracilis [sensu Lang, 1948] EXP1 P1 es más de dos veces la longitud de EXP2 P1. Eupelte setacauda difiere de E. cubensis sp. nov. en la armadura del BENP: EXP P5, siendo 4:5 en E. setacauda y 5:6 en E cubensis sp. nov.

\section{Género Peltidium Philippi, 1838}

\section{Peltidium antillensis sp. nov.}

Figuras 4-6

Descripción. Largo máximo, sin incluir la setas de la rama caudal, $950 \mu \mathrm{m}$. Ancho máximo $625 \mu \mathrm{m}$. Cefalotórax con epímeros puntiagudos. Los segmentos del 2 al 5 son más estrechos que el cefalotórax, los epímeros de estos segmentos están bien desarrollados y se encuentran expandidos lateralmente. Rostro amplio dirigido ventralmente.

Segmentos abdominales retraídos dentro del segmento genital, no observables en vista dorsal. Rama caudal cuatro veces más larga que ancha con seis setas. Anténula con siete artejos, artejos 3 y 4 con estetascos. Exopodito de la antena con dos artejos, artejo 1 con una seta lateral y artejo 2 con tres setas distales. Endopodito con dos artejos, artejo 1 con una seta, borde externo del artejo 2 con dos setas, una cercana a la base y otra cercana a su porción distal, que tiene cinco setas, 4 de ellas geniculadas.

Precoxopodito de la mandíbula con borde cortante grande y aplanado armado con siete dientes redondeados y fuertes y una seta distal. Palpo mandibular con dos artejos, artejo 1 desarmado y tres veces más largo que el artejo 2, el cual presenta una seta lateral y cuatro setas distales. Maxílula con siete dientes y dos enditos; coxopodito representado por un pequeño endito con dos setas; basipodito con dos lóbulos con cuatro y una seta respectivamente.

Maxila con tres enditos, el distal es el más largo con tres setas, el central solo una y el proximal tiene dos. Basipodito termina en un proceso puntiagudo y una seta, borde externo con tres setas e interno desarmado. Basipodito del maxilípedo con una seta, endopodito casi cuatro veces tan largo como ancho, borde externo con un pequeño grupo de setas, borde interno con hileras de pequeñas y finas setas. Garra curvada, casi de la misma longitud de endopodito con una pequeña seta en su base.

Pata 1 con coxopodito alargado con el borde interno y externo con numerosas setas. Basipodito con una seta en el borde externo. Exopodito con tres artejos, artejo 2 es el más largo de los tres. Artejo 1 con una seta en su borde externo, artejo 2 con dos setas una en el borde externo y otra en el interno, ambas cercanas al extremo distal, artejo 3 casi tan largo como ancho con dos setas y dos garras distales . Endopodito con dos artejos, artejo 1 más largo que ancho con ambos bordes con numerosas sétulas y una seta en su borde interno, artejo 2 con ambos bordes con numerosas sétulas con una seta subdistal y dos distales. 

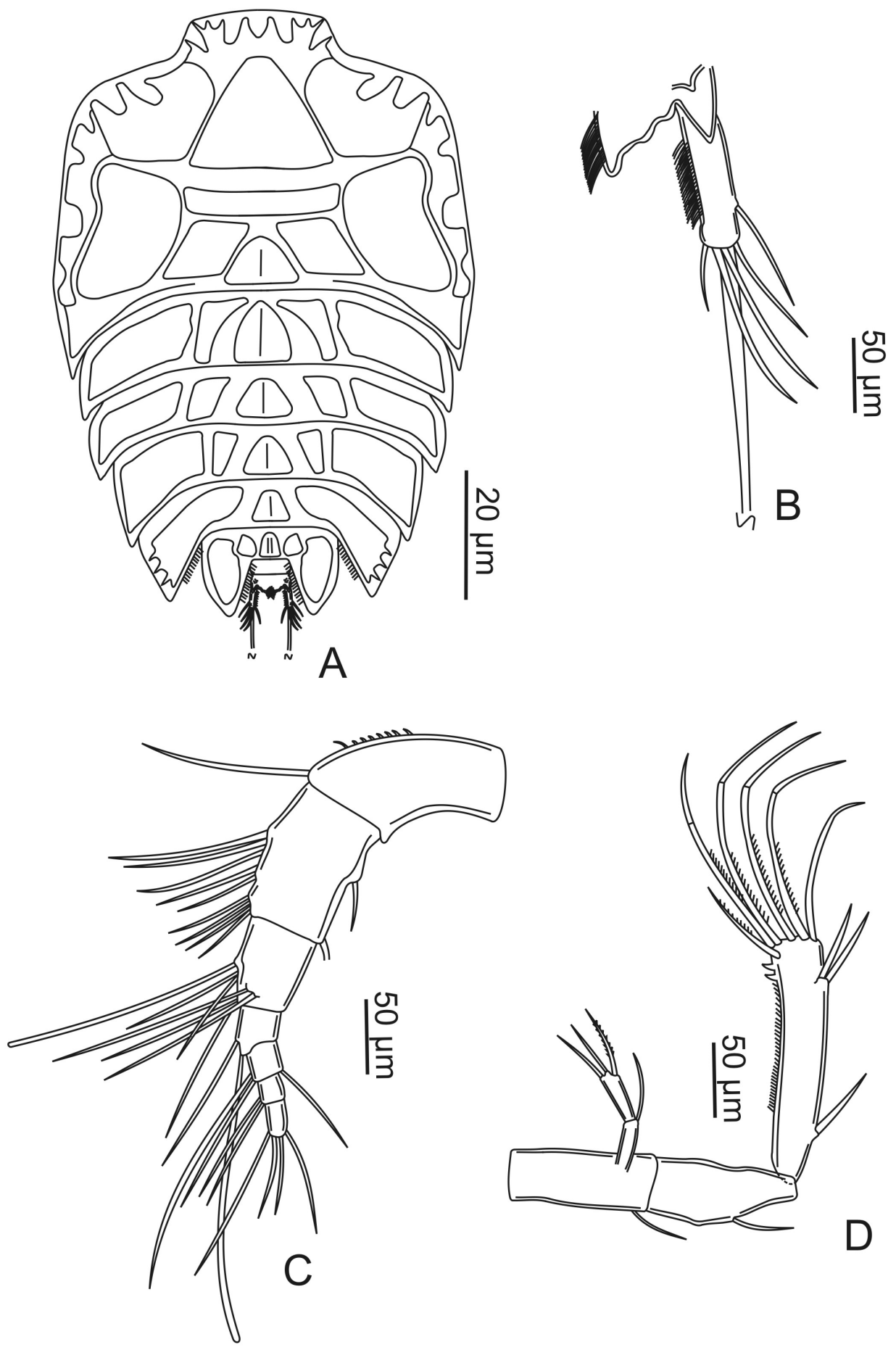

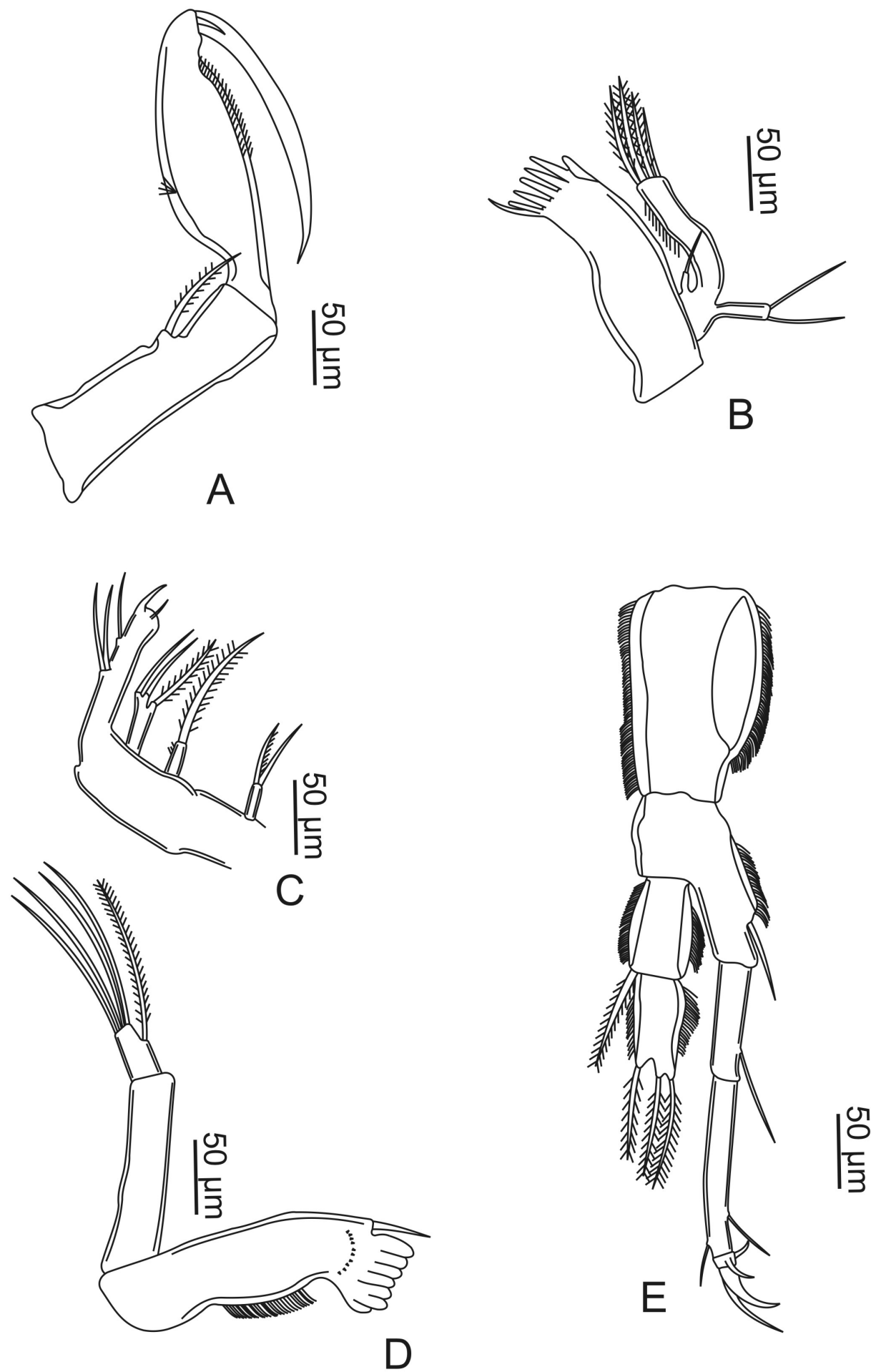
Basoendopodito de la pata 5 reducido con dos setas en su borde interno y una en el borde externo. Exopodito 5 veces más largo que ancho con una seta y dos espinas fuertes en su margen externo y una terminal y dos setas en el margen interno distal.

Patas 2-4 birramosas con tres artejos en cada rama. Coxas pequeñas y basipoditos alargados transversalmente con una seta lateral. El patrón de espinas y setas se muestra a continuación:

\begin{tabular}{lll}
\hline Pata & Exopodito & Endopodito \\
\hline P2 & $1 ; 1 ; 2,2,3$ & $1 ; 2 ; 1,2,0$ \\
P3 & $1 ; 1 ; 3,2,3$ & $1 ; 2 ; 2,2,0$ \\
P4 & $1 ; 1 ; 3,2,2$ & $1 ; 1 ; 2,2,0$ \\
\hline
\end{tabular}

Tipos. Holotipo. Hembra no ovígera. CUBA. Colectada en Loma del Puerto, Cayo Coco, norte de la provincia de Villa Clara $\left(22^{\circ} 33^{\prime} 22^{\prime \prime} \mathrm{N}\right.$; $78^{\circ} 24^{\prime} 55^{\prime}$ W); el 14.ii.2009 a $3 \mathrm{~m}$ de profundidad; col. C. Varela. Depositado en la Colección Natural Marina del Acuario Nacional de Cuba ANC.07.2.1.059. Paratipos: dos hembras no ovígeras. Los mismos datos del holotipo ANC.07.2.1.060.

Etimología. La especie fue nombrada antillensis, por la zona geográfica en que fue encontrada que es también conocida como Las Antillas.

Comentarios. Las conspicuas bandas quitinosas que se anastomosan a lo largo de los segmentos corporales son muy útiles para la separación de los diferentes géneros de la familia Peltidiidae. En un intento por clarificar la identidad e identificar las especies que en ese momento se incluían dentro del género Peltidium. Nicholls (1941) presentó una clave para las especies dentro de ese género. Algunas de las especies presentadas en el trabajo referido fueron posteriormente consideradas como no válidas por Lang (1948), quien también presentó el primer análisis comprensivo del género y notó la necesidad de prestar especial atención a la estructura y la setotaxia de la P1 y de la P5 para diferenciar las 15 especies que en aquel tiempo se atribuían al género Peltidium. Dos décadas más tarde, Geddes (1968) sugiere tres grupos de especies basados en la estructura general de la P5, Wells y Rao (1987) encuentran alta variabilidad intraespecífica en muchos de los caracteres usados en la separación de las especies. Los posteriores análisis del género se han visto obstaculizados por la pérdida del material tipo de varias de las especies cuya descripción original carece de los detalles necesarios para estudios más profundos.

En estos momentos el género Peltidium consiste de 27 especies (Varela, 2005 y Wells, 2007), muchas de las cuales muestran una distribución tropical y subtropical, habiendo solo dos especies conocidas para latitudes templadas. Más de la mitad de las especies conocidas han sido halladas en los Océanos Índico y Pacífico, mientras que las restantes especies se han encontrado en Europa y en el Atlántico Occidental Tropical. Peltidium antillensis sp. nov., presenta la pata cinco (P5) estiliforme, con el ENP muy reducido, el EXP mucho más largo que ancho y los últimos artejos de los ENP P2-P4 con la fórmula 3, 4, 4. Estos caracteres solo son compartidos por P. ovale Thompson I. C. \& Scott A., 1903, del Océano Índico; P. simplex Nicholls, 1941, de Australia; $P$. perturbatum Geddes, 1968, de Bahamas y P. proximus Varela, 2005, de Cuba. En Peltidium perturbatum y $P$. proximus los epímeros del cefalotórax llegan hasta la mitad del segmento 3, mientras que en $P$. antillensis sp. nov., estos epímeros solo llegan hasta la porción proximal del segmento 2 . En $P$. ovale y $P$. simplex el exopodito de la pata 5 tiene solo 5 setas, mientras que en $P$. antillensis sp. nov., tiene 6 setas. 

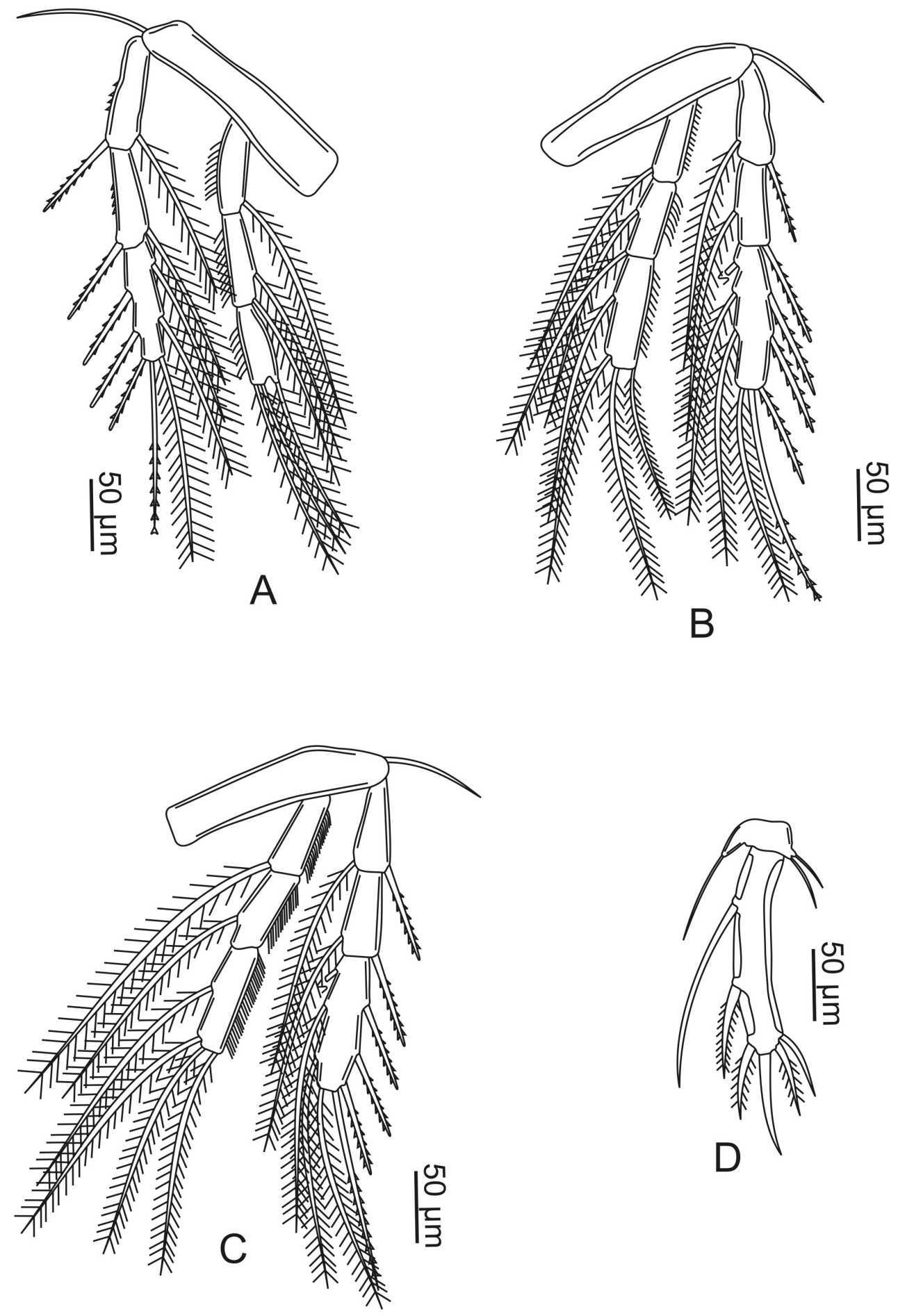

Figura 6. Peltidium antillensis sp. nov. A. Pata 2; B Pata 3; C. Pata 4 y D. Pata 5. 


\section{LITERATURA CITADA}

Boxshall, G. A. y S. H. Halsey. 2004. An Introduction to Copepod Diversity. The Ray Society, London 166, 1: 1-421.

Dahms, H. U. 1992. Peltidiidae (Copepoda, Harpacticoida) from the Weddell Sea (Antarctica). Zoologica Scripta 21 (2): 181-195.

Geddes, D. C. 1968. Marine biological investigations in the Bahamas. 7. Harpacticoid copepods belonging to the families Porcellidiidae Sars, Peltidiidae Sars and Tegastidae Sars. Sarsia 35: $9-56$.

Gomez, S y C. Varela 2013. New species of Altheuta Baird (Harpacticoida: Peltidiidae) from north-western Mexico. Journal of Natural History 47 (5-12): 313-328.

Hicks, G. R. F. 1982. Porcellidiidae and Peltidiidae (Copepoda: Harpacticoida) from the marine algae of ST. Croix Island, Algoa Bay, South Africa. Zoological Journal of the Linnean. Society 75: 49-90.

Lang, K. 1948. Monographie der Harpacticiden. Håkan Ohlssons Lund Sweden, Vol.I y II. 1682 p.

Nicholls, A. G. 1941. Littoral Copepoda from South Australia. I- Harpacticoida. Records of South Australia Museum 6: 381-427.

Varela, C. 2005. Una nueva especie de Peltidium (Copepoda: Harpacticoida), de Cuba. Solenodon 5: $1-5$.

Varela, C. 2010. Descripción de los machos de Peltidium nichollsi Geddes, 1968 y P. proximus, 2005 (Copepoda, Harpacticoida, Peltidiidae). Novitates Caribaea 3: 44-47.

Varela, C., S. Castellanos y L. Hernández. 2008. Registros nuevos de invertebrados (Cnidaria y Crustacea) para Cuba. Cocuyo 17: 12-14.

Wells, J. B. J. 2007. An annotated checklist and keys to the species of Copepoda Harpacticoida (Crustacea). Zootaxa 1568: 1-872.

Wells, J. B. J. y G. C. Rao. 1987. Littoral Harpacticoida (Crustacea: Copepoda) from Andaman and Nicobar Islands. Memoirs of the Zoological Survey of India 16 (4): 1-385.

[Recibido: 02 de octubre del 2012. Aceptado para publicación: 01 de febrero del 2013] 